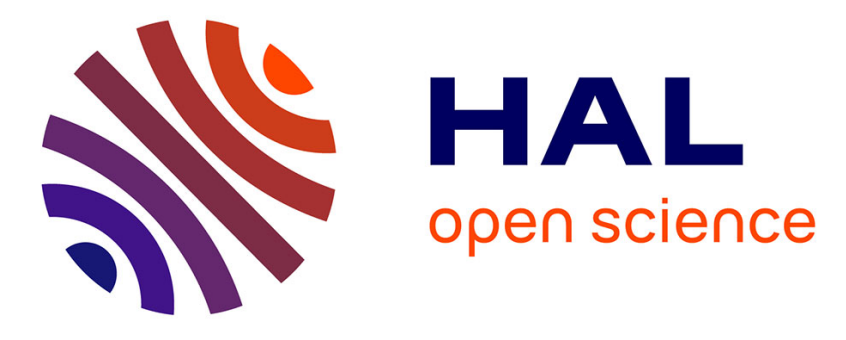

\title{
Integral backstepping control for trajectory tracking of a hybrid vehicle
}

\author{
J Colmenares-Vázquez, Nicolas Marchand, Pedro Castillo, Jose-Ernesto \\ Gomez-Balderas, Jonatan Álvarez-Muñoz, Jose Juan Téllez-Guzmán
}

\section{- To cite this version:}

J Colmenares-Vázquez, Nicolas Marchand, Pedro Castillo, Jose-Ernesto Gomez-Balderas, Jonatan Álvarez-Muñoz, et al.. Integral backstepping control for trajectory tracking of a hybrid vehicle. ICUAS 2015 - International Conference on Unmanned Aircraft Systems, Jun 2015, Denver, United States. pp.209-217, 10.1109/ICUAS.2015.7152293 . hal-01211121

\section{HAL Id: hal-01211121 \\ https://hal.science/hal-01211121}

Submitted on 6 Oct 2015

HAL is a multi-disciplinary open access archive for the deposit and dissemination of scientific research documents, whether they are published or not. The documents may come from teaching and research institutions in France or abroad, or from public or private research centers.
L'archive ouverte pluridisciplinaire HAL, est destinée au dépôt et à la diffusion de documents scientifiques de niveau recherche, publiés ou non, émanant des établissements d'enseignement et de recherche français ou étrangers, des laboratoires publics ou privés. 


\title{
Integral Backstepping Control for Trajectory Tracking of a Hybrid Vehicle
}

\author{
J. Colmenares-Vázquez, N. Marchand, P. Castillo, J.E. Gómez-Balderas, \\ J.U. Alvarez-Muñoz, J.J. Téllez-Guzmán
}

\begin{abstract}
This article is focused on the trajectory tracking using a hybrid terrestrial aerial vehicle. An integral backstepping control is proposed for the UAV vehicle mode. In addition, a nested saturation control is developed and applied to regulate the position of the cart vehicle. These control laws are validated by simulations and some experimental results on position control was performed by applying the techniques aforementioned.
\end{abstract}

\section{SYSTEM DESCRIPTION}

In this work control laws are developed for trajectory tracking of a hybrid terrestrial aerial vehicle. These kinds of vehicles have the advantage to be used as a flying vehicle or as a cart depending on the situation. Some situations may be when the vehicle find an obstacle and it has to take the more convenient mode of operation to overcome or to avoid the obstacle. Controlling these hybrid vehicles becomes a challenge. It is necessary to design and implement control laws for the trajectory following in the air and over the floor. The control strategy has to generate a smooth transition when the drone is passing from air to floor or vice versa.

There are several works dedicated to path following with hexarotors and also for carts, see for example [1]-[3]. This work considers a particular hybrid vehicle : a mini-UAV that is converted in a cart by attaching to it two wheels without any additional motors as in Fig. 1. The orientation and position of the cart will be controlled by the yaw and pitch angles and by the thrust generated by its helices. Among its characteristics, the thrust direction can be inversed as a result of the pitch angle variation. Therefore, the cart-drone can move forward or backward depending on the sign of pitch angle. This hybrid vehicle in terrestrial mode can turn around $z$ axis. It is also a nonholonomic system because it is not capable to move on the wheels axis direction in terrestrial mode. For more references in cart control refer to [4]-[6].

*This work was supported in part by the CONACYT (Consejo Nacional de Ciencia y Tecnología) of the Mexican government and in part by GIPSA$\mathrm{LAB}$ in France

J. Colmenares Vázquez, N. Marchand, J.E.Gomez Balderas, J.U. Alvarez-Muñoz and J.J. Téllez-Guzmán are with GIPSA LAB, UMR5216 , France. josue.colmenares-vazquez, nicolas.marchand, jose-ernesto.gomez-balderas, Jonatan-Uziel.Alvarez-Munoz, jose-juan.tellez-guzman @gipsa-lab.fr

P. Castillo is with Laboratoire Heudiasyc, UMR7253, France castillo@hds.utc.fr
The model of the vehicle can be obtained by using quaternions, the Lagrangian or the Newton Euler approach. This work will use a simplified model that can be obtained by the last approach. For a detailed deduction of the model of a UAV or of a cart, see [7]-[9]. This simplified model considers a rigid body perfectly symmetric and with mass and rotation centers placed at the same point. It is not taken into account the helix flexibility of the mini-UAV. Additionally, the effect of battery discharge is neglected. Some techniques have been developed to compensate these dynamics not considered here, for instance those ones in [10]-[15]. The reader may also find useful to review [16]-[19] related to other control strategies based in LQR, PID, CLF and saturation functions.

The protoype used is a Flexbot hexarotor. This is a miniUAV, low cost, and not represent an hazard. Fig. 1 shows the drone at issue. The tools used for the implementation of the control were MATLAB ${ }^{\circledR}$, xPC Target and Vicon System.

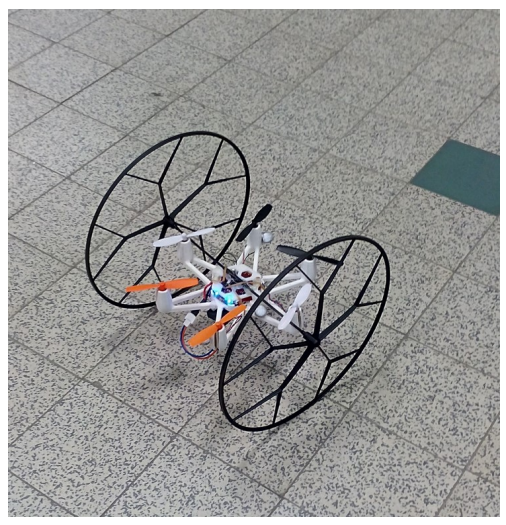

Fig. 1. Cart-drone used in the experiments

\section{UAV AND CART PROTOTYPE}

All experiments were performed in MOCA room. The MOCA room at GIPSA-LAB is a platform dedicated for the movement capture using several cameras, cf. [20]. The Flexbot hexarotor has a microprocessor ATMega32u4, a gyro and an accelerometer MPU6050 and a flight controller Microwii Copter. This mini drone can load until 12 grams in UAV mode. The drone was placed in MOCA room in order to get its 3D position and orientation angles by the VICON Tracker System. Once the position and orientation 
are passed to the control law and executed by xPC Target, the desired control laws are sent to Flexbot via Bluetooth LE. Finally, the Flexbot drone execute the orientation control programmed inboard.

Fig. 2 summarizes the control process. The parameters related to the two prototypes are shown in Tables I and II.

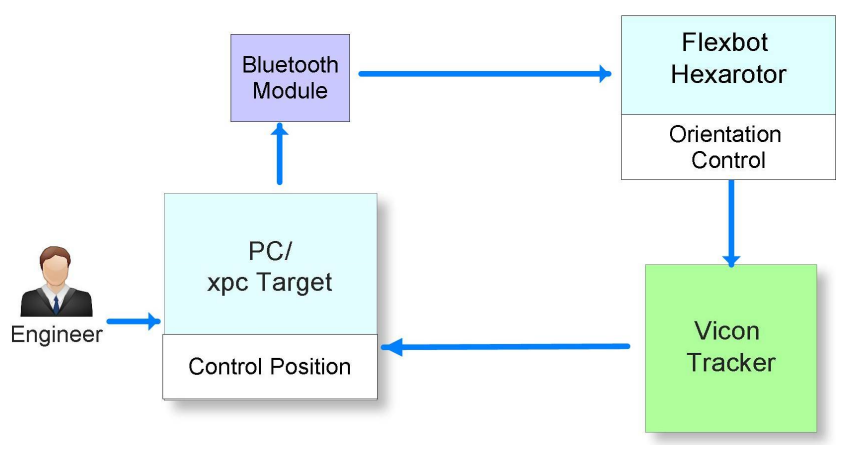

Fig. 2. Process flow used to control the hexarotor

TABLE I

HEXAROTOR PARAMETERS

\begin{tabular}{|c||c|}
\hline Parameter & Value \\
\hline \hline mass & $0.057 \mathrm{~kg}$ \\
\hline payload & $0.023 \mathrm{~kg}$ \\
\hline max diameter & $0.12 \mathrm{~m}$ \\
\hline helix length & $0.05 \mathrm{~m}$ \\
\hline
\end{tabular}

TABLE II

CART PARAmeters

\begin{tabular}{|c||c|}
\hline Parameter & Value \\
\hline \hline mass & $0.068 \mathrm{~kg}$ \\
\hline payload & $0.012 \mathrm{~kg}$ \\
\hline wheels diameter & $0.14 \mathrm{~m}$ \\
\hline
\end{tabular}

\section{UAV CONTROL ALGORITHM}

Our first approach is to propose a control law for the vehicle in aerial mode. In the following, the dynamic model and the control will be described.

\section{A. Mathematical Model}

The UAV is considered as a rigid body and the model is obtained from the Euler Newton approach. The system considered is show in Fig. 3:

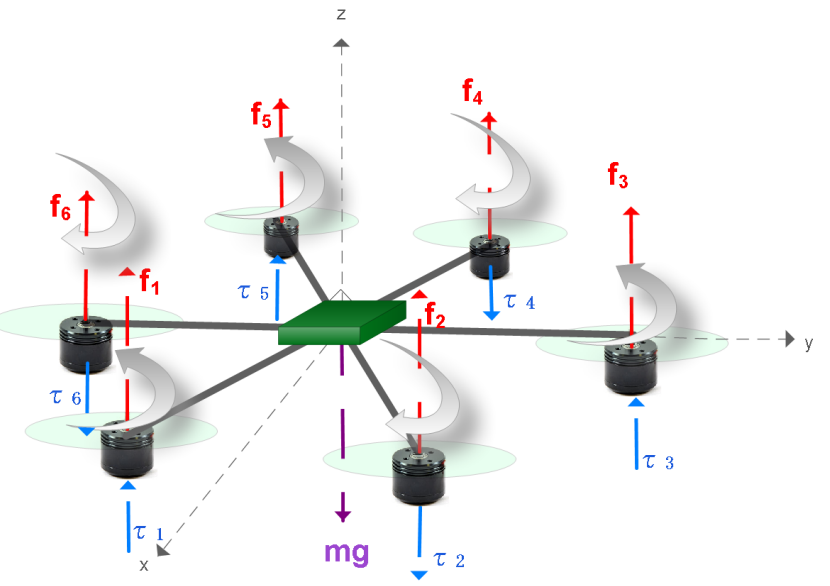

Fig. 3. Forces and torques acting on a simplified model of the hexarotor

The equations of the model are the following:

$$
\begin{aligned}
m \ddot{r} & =R F+F_{g} \\
\dot{\eta} & =B(\eta) \Omega \\
\mathbb{J} \dot{\Omega} & =\tau-\omega^{\times} \mathbb{J} \Omega
\end{aligned}
$$

where $m$ is the mass of drone and $r$ defines the position of mass center of the drone in the inertial system. $R$ describes the rotation matrix generated in the order yaw-pitch-roll. $F$ means the vector of thrust generated by the helices. $F_{g}$ represents the vector of gravity force. $\eta$ stands for the vector of Euler angles, $\Omega$ is the vector of angular velocities in the body frame. $B(\eta)$ represents the matrix relating the vector of angular velocities and the derivative of the Euler angles. $\mathbb{I}$ is the inertia matrix of the drone. $\omega^{\times}$means the skew symmetric matrix of angular velocity and $\tau$ defines the vector of torques applied to the vehicle.

\section{B. Control Law for Trajectory Following}

The approach used for the trajectory tracking is based in the Backstepping technique. To ensure the convergence to desired trajectory an integral part is added. The integral part helps to reduce the error of the tracking and adds a factor that improves the robustness when the parameters of the system are not well-known.

Let us define the position error:

$$
e_{r}=r-r_{r e f} \quad \Longrightarrow \quad \dot{e}_{r}=\dot{r}-\dot{r}_{r e f}=v-\dot{r}_{r e f}
$$

Let us propose a positive definite function to design a convenient velocity $v^{v}$ that ensures the convergence to the desired position.

$$
V_{L r}=\frac{1}{2}<\chi_{1}, K_{I r} \chi_{1}>+\frac{1}{2}<e_{r}, e_{r}>
$$

where $\langle *, *\rangle$ means the inner vectorial product, $K_{I r}$ is a positive diagonal constant matrix that will be used for tuning the control and

$$
\chi_{1}=\int_{0}^{t} e_{r} d \tau
$$


Therefore

$$
\dot{V}_{L r}=<\chi_{1}, K_{I r} e_{r}>+<e_{r}, \dot{e}_{r}>
$$

Now, taking the virtual velocity control as:

$$
v^{v}=\dot{r}_{r e f}-K_{I r} \chi_{1}-K_{r} e_{r}
$$

and thus,

$$
\left.\dot{V}_{L r}\right|_{v=v^{v}}=-<e_{r}, K_{r} e_{r}>\quad \leq 0 \quad \forall t \geq 0
$$

Let us define the velocity error:

$$
e_{v}=v-v^{v} \quad \Longrightarrow \quad \dot{e}_{v}=\ddot{r}-\dot{v}^{v}=\frac{1}{m} u-\dot{v}^{v}
$$

and let be the following positive definite function:

$$
V_{L v}=V_{L r}+\frac{1}{2}<e_{v}, e_{v}>
$$

thus

$$
\dot{V}_{L v}=\dot{V}_{L r}+<e_{v}, \dot{e}_{v}>
$$

and taking into account that $v=v^{v}+e_{v}$, the expression $\dot{V}_{L v}$ can be written as:

$$
\dot{V}_{L v}=-<e_{r}, K_{r} e_{r}>+<e_{r}, e_{v}>+<e_{v}, \dot{e}_{v}>
$$

Choosing the virtual control,

$$
u^{v}=m\left(\dot{v}-e_{r}-K_{v} e_{v}\right)
$$

It results that:

$$
\begin{aligned}
\left.\dot{V}_{L v}\right|_{u=u^{v}} & =-<e_{r}, K_{r} e_{r}>-<e_{v}, K_{v} e_{v}> \\
& \leq 0 \quad \forall t \geq 0
\end{aligned}
$$

where $K_{v}$ is a positive diagonal constant matrix that will be used for tuning the control. Keeping in mind that $u=R F+F_{g}$, where $R$ depends on Euler angles vector $\eta$. Hence, there exists a relationship between $u$ and $(\eta, F)$ that let us determine the value of $u$ at every time. Let us call $\eta_{r e f}$ and $F_{r e f}$ the Euler angles and the thrust, respectively, needed to generate $u=u^{v}$. Now, the following development will be focused in designing a torques vector capable of generating the desired vectors of Euler angles.

The thrust vector $F$ and weight vector $F_{g}$ are defined as follows:

$$
F=\left(\begin{array}{l}
0 \\
0 \\
f
\end{array}\right) \quad F_{g}=\left(\begin{array}{c}
0 \\
0 \\
-m g
\end{array}\right)
$$

where $g$ is the gravity acceleration and considered here as $9.81 \mathrm{~m} / \mathrm{s}^{2}$. The rotation matrix $R$ is obtained from Euler angles in the order yaw-pitch-roll and has the following expression:

$$
R=\left(\begin{array}{ccc}
c \psi c \theta & -s \psi c \phi+c \psi s \theta s \phi & s \psi s \phi+c \psi s \theta c \phi \\
s \psi c \theta & c \psi c \phi+s \psi s \theta s \phi & -c \psi s \phi+s \psi s \theta c \phi \\
-s \theta & c \theta s \phi & c \theta c \phi
\end{array}\right)
$$

where $s$. and $c$. mean $\cos (\cdot)$ and $\sin (\cdot)$ respectively. The yaw, pitch and roll angles are given by $\psi, \theta, \phi$ respectively.
The Euler angles vector and the force vector $u$ are defined as:

$$
\eta=\left(\begin{array}{c}
\psi \\
\theta \\
\phi
\end{array}\right) \quad u=\left(\begin{array}{l}
u_{x} \\
u_{y} \\
u_{z}
\end{array}\right)
$$

Then, from (1), (14), (15) and (16) it can be deduced the thrust and the Euler angles needed to generate the virtual control $u^{v}$. The $\psi_{\text {ref }}$ needed can be chosen arbitrarily or conveniently. $\theta_{\text {ref }}, \phi_{\text {ref }}$ and $f_{r e f}$ have the following expressions:

$$
\begin{aligned}
& \theta_{\text {ref }}=\arctan \left(\frac{u_{y} s \psi+u_{x} c \psi}{u_{z}+m g}\right) \\
& \phi_{\text {ref }}=\arctan \left(c \theta_{\text {ref }} \cdot \frac{u_{x} s \psi-u_{y} c \psi}{u_{z}+m g}\right) \\
& f_{\text {ref }}=\frac{u_{z}+m g}{c \theta_{\text {ref }} \cdot c \phi_{\text {ref }}}
\end{aligned}
$$

Define the Euler angles error as:

$$
\begin{aligned}
e_{\eta}=\eta-\eta_{\text {ref }} \quad \Longrightarrow \quad \dot{e}_{\eta} & =\dot{\eta}-\dot{\eta}_{\text {ref }} \\
& =B(\eta) \Omega-\dot{\eta}_{\text {ref }}
\end{aligned}
$$

The matrix $B(\eta)$ has the following form:

$$
B=\left(\begin{array}{ccc}
0 & s \phi / c \theta & c \phi / c \theta \\
0 & c \phi & -s \phi \\
1 & s \phi \cdot t \theta & c \phi \cdot t \theta
\end{array}\right)
$$

where $t$ means $\tan$. The matrix $B(\eta)$ is not singular if and only if $\cos (\theta) \neq 0$.

Let us propose the next positive definite function:

$$
V_{L \eta}=\frac{1}{2}<\chi_{2}, K_{I \eta} \chi_{2}>+\frac{1}{2}<e_{\eta}, e_{\eta}>
$$

where,

$$
\chi_{2}=\int_{0}^{t} e_{\eta} d \tau
$$

and $K_{I \eta}$ is a positive diagonal constant matrix that will be used for tuning the control. Thus,

$$
\dot{V}_{L \eta}=<\chi_{2}, K_{I \eta} e_{\eta}>+<e_{\eta}, \dot{e}_{\eta}>
$$

and by choosing the virtual angular velocity $\Omega^{v}$,

$$
\Omega^{v}=B^{-1}\left(\dot{\eta}_{r e f}-K_{I \eta} \chi_{2}-K_{\eta} e_{\eta}\right)
$$

with $K_{\eta}$ as a positive diagonal constant matrix, it yields

$$
\left.V_{L \eta}\right|_{\Omega=\Omega^{v}}=-K_{\eta}<e_{\eta}, e_{\eta}>\leq 0 \quad \forall t \geq 0
$$

Now, let us define the angular velocity error as:

$$
e_{\Omega}=\Omega-\Omega_{\eta} \quad \Longrightarrow \quad \dot{e}_{\Omega}=\dot{\Omega}-\dot{\Omega}_{\eta}
$$

and remember that,

$$
\Omega=\Omega_{\eta}+e_{\Omega} \quad \& \quad \dot{\Omega}=\mathbb{J}^{-1}\left(\tau-\omega^{\times} \mathbb{J} \Omega\right)
$$

Now, consider the following candidate Lyapunov function:

$$
V_{L \Omega}=V_{L \eta}+\frac{1}{2}<e_{\Omega}, e_{\Omega}>
$$


then,

$$
\dot{V}_{L \Omega}=\dot{V}_{L \eta}+<e_{\Omega}, \dot{e}_{\Omega}>
$$

therefore,

and by choosing,

$$
\tau=\omega^{\times} \mathbb{J} \Omega+\mathbb{J}\left(\dot{\Omega}_{\eta}-B^{T} e_{\eta}-K_{\Omega} e_{\Omega}\right)
$$

it yields that,

$\dot{V}_{L \Omega}=-<e_{\eta}, K_{\eta} e_{\eta}>-<e_{\Omega}, K_{\Omega} e_{\Omega}>\leq 0 \quad \forall t \geq 0$ (33)

with $K_{\Omega}$ as a positive diagonal constant matrix used for control purposes.

\section{CONTROL STRATEGY FOR THE CART VEHICLE}

A control algorithm for the cart vehicle to follow a desired trajectory is developed in this section. The vehicle inclination correspond to pitch angle and the cart orientation to yaw angle. The control law will focus in the pitch and yaw in order to make the trajectory tracking. The desired roll angle is zero because the wheels will stay in touch with the floor. Additionally, the thrust generated by helices has to be limited in order to avoid the take off. In this work, the floor is considered as flat.

\section{A. Mathematical Model}

The cart-drone is depicted in Fig. 4. The body frame is represented by $\left(x_{b}, y_{b}, z_{b}\right)$ The model of the cart-drone is obtained from the model of a rigid hexarotor. It is considered only the projection on $x y$-plane. Therefore, from Fig. 4, we can deduce that

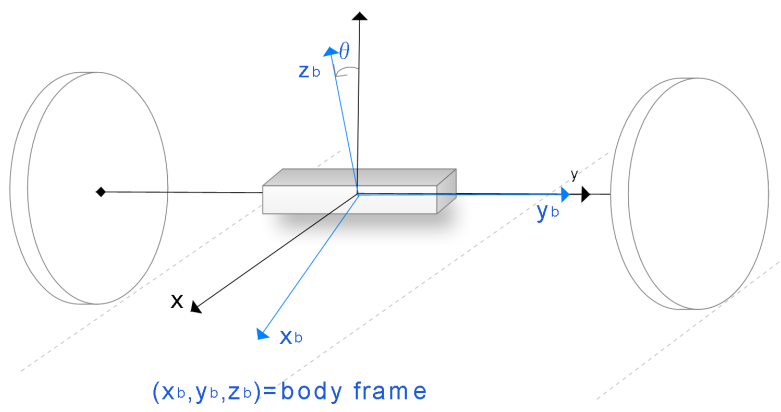

Fig. 4. Cart diagram

$$
M \frac{d^{2}}{d t^{2}}\left(\begin{array}{l}
x \\
y
\end{array}\right)=\left(\begin{array}{c}
\cos \psi \\
\sin \psi
\end{array}\right) \sin \theta \cdot f
$$

where $\psi$ is the yaw angle and $\theta$ is the pitch angle. Equation (34) can be written as:

$$
M \frac{d^{2}}{d t^{2}}\left(\begin{array}{l}
x \\
y
\end{array}\right)=R_{x y}\left(\begin{array}{l}
\nu \\
0
\end{array}\right)
$$

where $R_{x y}$ stands for the rotation matrix in $x y$-plane and $\nu$ is the projection of thrust over $x y$-plane. Then, the expressions for $R_{x y}$ and $\nu$ are:

$$
R_{x y}=\left(\begin{array}{cc}
\cos \psi & -\sin \psi \\
\sin \psi & \cos \psi
\end{array}\right) \quad \nu=\sin \theta \cdot f
$$

The model corresponding to the cart-drone attitude is the following:

$$
\begin{aligned}
\dot{\eta} & =B(\eta) \Omega \\
\mathbb{d} \dot{\Omega} & =\tau-\omega^{\times} \mathbb{J} \Omega+\tau_{\text {floor }}
\end{aligned}
$$

where $\tau_{\text {floor }}$ represents torque exercised by the floor when the cart wheels make contact with it. Notice that the floor is supposed horizontal and the wheels need to be in contact permanently with it, thus the desired roll angle will be $\phi_{d}=0$. Furthermore, the torques, caused by the floor when the cart wheels touch it, will bring about the cart stay in contact with the floor.

\section{B. Control Law for Trajectory Following}

The control law proposed here is separated in two parts. The first part of the strategy consists in the design of a saturation control in order to obtain a desired force such that the vehicle can perform the tracking. Similarly to the strategy followed in the control design for the vehicle as a $\mathrm{UAV}$, the attitude part is modeled by means of the Euler angles and these ones keep a direct relationship with the desired force. Hence, there exists a local bijective mapping between the angles and the required force. Afterwards, the backstepping control developed for the attitude part in the previous section is used in order to generate the desired saturation control.

The saturation control is desired for generating a lesser aggressive response by limiting the velocity of the vehicle and as well it will help us to delimit the required thrust and the maximum desired pitch. The backstepping technique is used in order to produce the desired force that ensure the trajectory tracking. The position model showed in (34) can be rewritten as

$$
\begin{aligned}
\dot{r} & =v \\
M \dot{v} & =u
\end{aligned}
$$

with

$$
r=\left(\begin{array}{l}
x \\
y
\end{array}\right), r_{d}=\left(\begin{array}{l}
x_{d} \\
y_{d}
\end{array}\right), u=\left(\begin{array}{c}
\cos \psi \\
\sin \psi
\end{array}\right) \sin \theta \cdot f
$$

The desired trajectory $r_{d}$ can be included in the model, thus the model is rewritten as:

$$
\begin{array}{ccc}
\dot{e}_{r} & = & p \\
m \dot{p} & = & u-m \ddot{r}_{d}
\end{array}
$$

where

$$
\begin{aligned}
e_{r} & =r-r_{d} \\
p & =v-\dot{r_{d}}
\end{aligned}
$$


The saturation function is defined as

$$
\sigma_{M}(s)=\left\{\begin{aligned}
M & \text { if } s \geq M \\
s & \text { if }|s|<M \\
-M & \text { if } s \leq-M
\end{aligned}\right.
$$

where $s$ is a scalar and $M>0$. In the following, it will be developed the control law corresponding to the $x$ coordinate.

Consider $u, p, e_{r}$ and $r_{d}$ as vectors with two components:

$$
\begin{gathered}
u=\left(\begin{array}{l}
u_{x} \\
u_{y}
\end{array}\right) p=\left(\begin{array}{l}
p_{x} \\
p_{y}
\end{array}\right) \\
e_{r}=\left(\begin{array}{l}
e_{x} \\
e_{y}
\end{array}\right) r_{d}=\left(\begin{array}{l}
r_{d x} \\
r_{d y}
\end{array}\right)
\end{gathered}
$$

Propose

$$
u_{x}=m \ddot{r}_{d x}-m K_{1 x} \sigma_{M_{1 x}}\left(K_{p x} p_{x}+K_{1 x}^{-1} K_{2 x} \sigma_{M_{2 x}}\right)
$$

with $M_{1 x} \geq K_{1 x}^{-1} K_{2 x} M_{2 x}$ and $K_{1 x}, M_{1 x}, K_{p x}, K_{2 x}$ and $M_{2 x}$ ar positive constant used for tuning the saturation control. Therefore, the second equation of (40) becomes:

$$
\dot{p}_{x}=-K_{1 x} \sigma_{M_{1 x}}\left(K_{p x} p_{x}+K_{1 x}^{-1} K_{2 x} \sigma_{M_{2 x}}\right)
$$

Then, there exists a time $T_{1}$ such that:

$$
\left|K_{p x} p_{x}\right| \leq K_{1 x}^{-1} K_{2 x} M_{2 x} \quad \forall t \geq T_{1}
$$

Taking

$$
M_{1 x} \geq 2 K_{1 x}^{-1} K_{2 x} M_{2 x}
$$

the saturation $\sigma_{M_{1 x}}$ results in:

$$
\sigma_{M_{1 x}}(\cdot)=K_{p x} p_{x}+K_{1 x}^{-1} K_{2 x} \sigma_{M_{2 x}} \quad \forall t \geq T_{1}
$$

which leads to (50)

$$
\dot{p}_{x}=-K_{1 x} K_{p x} p_{x}-K_{2 x} \sigma_{M_{2 x}} \quad \forall t \geq T_{1}
$$

Now, proposing another variable

$$
q_{x}=K_{1 x} K_{p x} e_{x}+p_{x}
$$

and by differentiating this variable it yields:

$$
\dot{q_{x}}=-K_{2 x} \sigma_{M_{2 x}}
$$

and by choosing $\sigma_{M_{2 x}}=\sigma_{M_{2 x}}\left(K_{q x} q_{x}\right)$ it results:

$$
\dot{q_{x}}=-K_{2 x} \sigma_{M_{2 x}}\left(K_{q x} q_{x}\right)
$$

with $K_{q x}$ as a positive constant used for tuning the controller. From (53) it is deduced that $q_{x}$ converges to 0 and by consequence

$$
p_{x} \rightarrow-K_{1 x} K_{p x} e_{x} \quad \text { as } \quad \mathrm{t} \rightarrow \infty
$$

Using this result in the first equation of (40), it yields

$$
e_{x} \rightarrow 0
$$

and from (54)

$$
p_{x} \rightarrow 0
$$

Therefore, the complete desired input, in order to make converge $e_{x}$ to 0 , will have the expression:

$u_{x}=m \ddot{r}_{d x}-m K_{1 x} \sigma_{M_{1 x}}\left(K_{p x} p_{x}+K_{1 x}^{-1} K_{2 x} \sigma_{M_{2 x}}\left(K_{q x} q_{x}\right)\right)$ (57)

In a similar way, the desired input $u_{y}$ which will make converge the $e_{y}$ to 0 has the corresponding expression:

$u_{y}=m \ddot{r}_{d y}-m K_{1 y} \sigma_{M_{1 y}}\left(K_{p y} p_{y}+K_{1 y}^{-1} K_{2 y} \sigma_{M_{2 y}}\left(K_{q y} q_{y}\right)\right)$ (58)

where $K_{1 y}, M_{1 y}, K_{p y}, K_{1 y}, K_{2 y}, M_{2 y}, K_{q y}$ are constant used for tuning the controller. The variable $q_{y}$ is defined in a similar way as in (51). Additionally, the system represented by (34) must satisfy the constraint:

$$
f \cos \theta \leq m g
$$

Now, it is necessary to find the desired couple $\left(\nu_{d}, \psi_{d}\right)$ that generates the virtual force $u_{d}=\left[\begin{array}{ll}u_{x} & u_{y}\end{array}\right]^{T}$ obtained in (57) and (58). Thus, from (34), the following equality is deduced:

$u_{x} \sin \psi_{d}-u_{y} \cos \psi_{d}=0 \Longrightarrow \sqrt{u_{x}^{2}+u_{y}^{2}} \sin \left(\psi_{d}+\zeta\right)=0$

There will be two possible solutions for $\psi_{d}$, namely,

$$
\begin{aligned}
\psi_{d} & =-\zeta \\
\psi_{d 1} & =\arg \left(u_{x}+i \cdot u_{y}\right)
\end{aligned}
$$

and the other possible solution is given by

$$
\psi_{d 2}=\arg \left(-u_{x}-i \cdot u_{y}\right)
$$

These two equations represent the same direction of vector $u_{d}$, and therefore the drone must to take the closest one to its yaw angle $\psi$. The direction of the thrust can be changed by means of pitch angle $\theta$ to ensure the movement in the correct sense. This situation is described in Fig. 5.

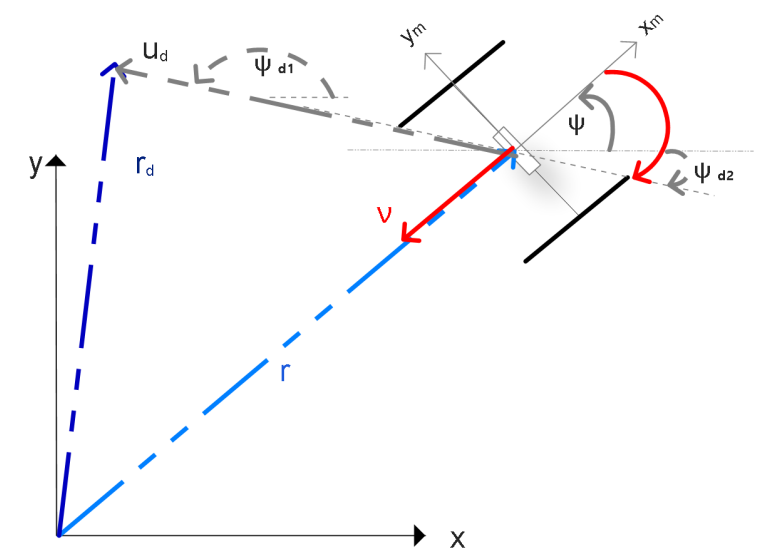

Fig. 5. Desired yaw angle for minimal effort

The goal is to reach the desired direction with the minimal effort. The closest angle satisfy $\cos \left(\psi-\psi_{d}\right) \geq 0$. Once the closest desired yaw angle is known, the expression for this angle will be rewritten in such a way that the cart will do 
the minimal effort to reach the desired direction. From (35), the following equality holds:

$$
R_{x y}{ }^{T} u_{d}=\left(\begin{array}{c}
\nu_{d} \\
0
\end{array}\right)
$$

Accordingly,

$$
u_{x} \cos \psi_{d}+u_{y} \sin \psi_{d}=\nu_{d}
$$

then,

$$
\sin \theta_{d}=\frac{u_{x} \cos \psi_{d}+u_{y} \sin \psi_{d}}{f}
$$

which can be simplified as:

$$
\sin \theta_{d}= \pm \frac{\sqrt{u_{x} 2+u_{y}^{2}}}{f}
$$

The sign depends on which $\psi_{d}$ is taken. It will be positive if (60) is chosen or negative if (61) is taken. The next step is to determine the thrust $f$. The thrust may be chosen arbitrarily but it must satisfy the conditions in the inequality (65), deduced from (34) and (63), in order to be capable of generate the required force $u_{d}$ and the pitch angle $\theta_{d}$.

$$
f \geq \sqrt{u_{x}^{2}+u_{y}^{2}}
$$

Taking,

$$
f=\frac{K_{\min }+\rho \sqrt{u_{x}^{2}+u_{y}^{2}}}{K_{f}+\cos \left(\psi-\psi_{d}\right)}
$$

where $K_{\min }, K_{f}$ and $\rho$ are positive constant. If $\rho$ is chosen as:

$$
\rho \geq K_{f}+1
$$

thus, (66) will satisfy (65). The factor $K_{f}+\cos \left(\psi-\psi_{d}\right)$ will allow the cart to prioritize finding the desired $\psi_{d}$ and afterwards, the cart will move to desired trajectory with the pitch angle determined by (63) but it is necessary to take $K_{f}<1$. By supposing $u_{x}=0, u_{y}=0, \psi=\psi_{d}$, the thrust given by

$$
\frac{K_{\min }}{K_{f}+\cos \left(\psi-\psi_{d}\right)}
$$

will let the cart to use this thrust to compensate the dynamics not taken into account in the mathematical model, e.g., the gravity center of cart is not perfectly placed at the rotation center. This expression allows to have a rotation according to the $K_{\min }$ value even when the drone has reached the desired position. The expression (66) must satisfy the condition in (59). In order to do this, it is sufficient that,

$$
\frac{K_{\min }+\rho \sqrt{\left(u_{x}\right)_{\text {max }}^{2}+\left(u_{y}\right)_{\text {max }}^{2}}}{K_{f}} \leq m g
$$

with $0<K_{f}<1$. It follows,

$$
\sqrt{\left(u_{x}\right)_{\max }^{2}+\left(u_{y}\right)_{\max }^{2}} \leq \frac{m g \cdot K_{f}-K_{\min }}{\rho}
$$

Since,

$$
|u| \leq m\left|\ddot{r}_{d}\right|_{\max }+m \sqrt{\left(K_{1 x} M_{1 x}\right)^{2}+\left(K_{1 y} M_{1 y}\right)^{2}}
$$

and if

$$
\begin{aligned}
m\left|\ddot{r}_{d}\right|_{\text {max }} & +m \sqrt{\left(K_{1 x} M_{1 x}\right)^{2}+\left(K_{1 y} M_{1 y}\right)^{2}} \\
& \leq \frac{m g \cdot K_{f}-K_{\text {min }}}{\rho}
\end{aligned}
$$

Then, the inequality in (70) still holds. Therefore,

$$
\sqrt{\left(K_{1 x} M_{1 x}\right)^{2}+\left(K_{1 y} M_{1 y}\right)^{2}} \leq \frac{g \cdot K_{f}-\left(K_{\min } / m\right)}{\rho}
$$

In order to simplify the previous expression, the following assumption is taken

$$
K_{1 x} M_{1 x}=K_{1 y} M_{1 y}=K_{1} M_{1}
$$

hence,

$$
K_{1} M_{1} \leq \frac{g \cdot K_{f}-\left(K_{\min } / m\right)}{\sqrt{2} \rho}-\frac{\left|\ddot{r}_{d}\right|_{\max }}{\sqrt{2}}
$$

Regarding the maximum desired pitch angle, from (64) and (66), it results that:

$$
\left|\theta_{d}\right|_{\text {max }} \leq \sin ^{-1}\left(\frac{1+K_{f}}{\rho}\right)
$$

For the attitude control, a backstepping control is proposed. The desired roll angle will be $\phi_{d}=0$, the desired pitch angle is given by (63), the desired yaw angle $\psi_{d}$ is given by (60) and (61) and the desired thrust is given by (66).

\section{SIMULATION AND EXPERIMENTAL RESULTS}

The integral backstepping control for the UAV and the saturation control for the cart proposed in previous sections were validated by simulations. The backstepping control was compared its performance of the standard backstepping with respect to the integral backstepping by making a circle at 1 meter over the floor. Likewise, the simulation corresponding to the cart was carried out in order to test the performance when the cart is tracking a circle over the floor.

\section{A. Control applied to UAV with a higher mass and inertia matrix}

The following parameters used in the UAV simulations were:

$$
\begin{array}{ll}
K_{I}=\operatorname{diag}(1,1,1) & K_{r}=\operatorname{diag}(1,1,1) \\
m=57 \mathrm{gr} & g=9.81 \mathrm{~m} / \mathrm{s} 2 \\
K_{\eta}=\operatorname{diag}(4,4,4) & K_{\Omega}=\operatorname{diag}(8,8,8) \\
J_{x x}=J_{y y}=8.833 \mathrm{gr} \cdot m 2 & J_{z z}=0.62993 \mathrm{gr} \cdot \mathrm{m} 2 \\
K_{v}=\operatorname{diag}(4,4,4) & K_{I \eta}=\operatorname{diag}(0.01,0.01,0.01) \\
J=\operatorname{diag}\left(J_{x x}, J_{y y}, J_{z z}\right) &
\end{array}
$$

In the first simulation, the UAV mass was 0.075 grams and the inertia matrix was considered as 1.5 times its nominal value. The performance of standard backstepping is shown in Figs. 6, 7. As shown in the corresponding figures, the standard backstepping control is not quite robust face to bigger mass. The performance of integral backstepping is depicted in the Figs. 8, 9, 10, 11. 


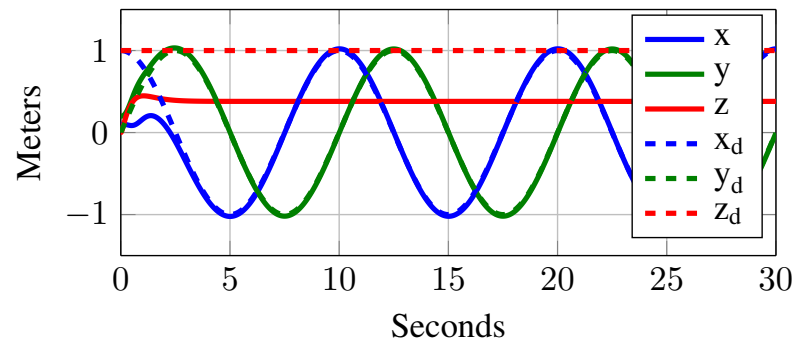

Fig. 6. Mini-UAV position under standard backstepping control with bigger mass and inertia matrix than modeled

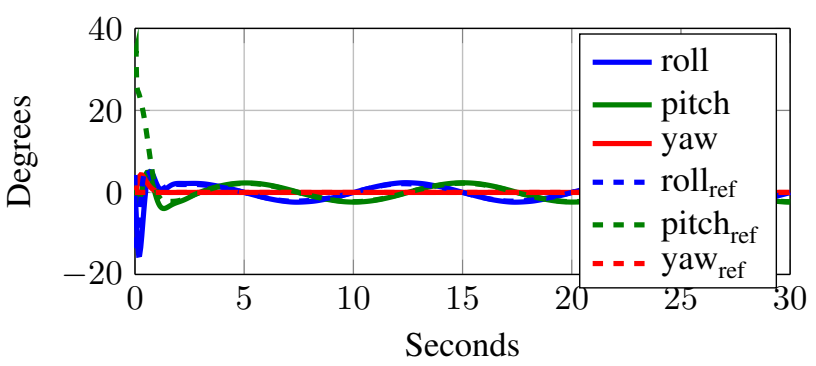

Fig. 7. Euler angles of drone using standard backstepping control with bigger mass and inertia matrix than modeled

In general, the integral backstepping will help to compensate the uncertainties in the dynamical model and will make more robust the control law face to little perturbations.

\section{B. Experimental results in position control of $U A V$}

In MOCA room at GIPSA-LAB was tested the integral backstepping in order to make a position control of an hexarotor Flexbot. Figs. 12, 13, and 14 show the first results obtained. There are dynamics not considered as the battery discharge speed, the mass center is not perfectly in the rotation center of the vehicle, the drone is considered as a perfect rigid body. The total effect of these dynamics not modeled is compensated by the integral backstepping.

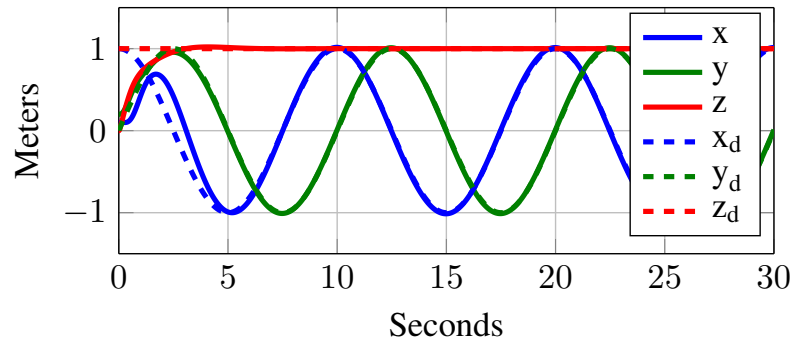

Fig. 8. Mini-UAV position response when using integral backstepping controller

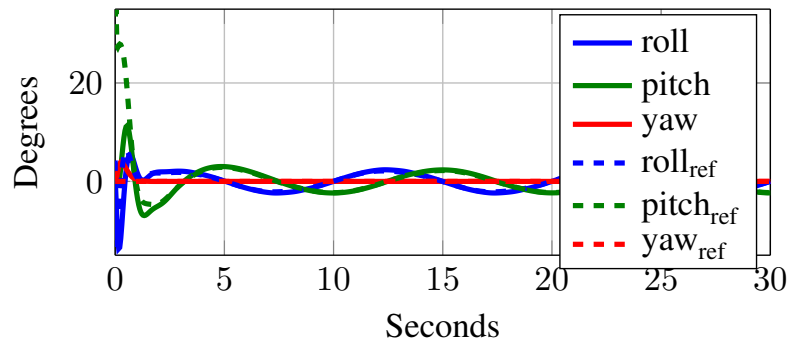

Fig. 9. Euler angles of UAV under integral backstepping control with bigger mass and inertia matrix than modeled

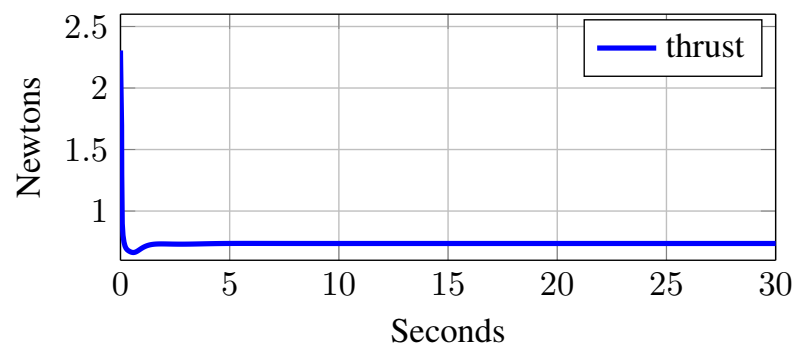

Fig. 10. Thrust generated by integral backstepping control when supposing bigger mass and inertia matrix than modeled

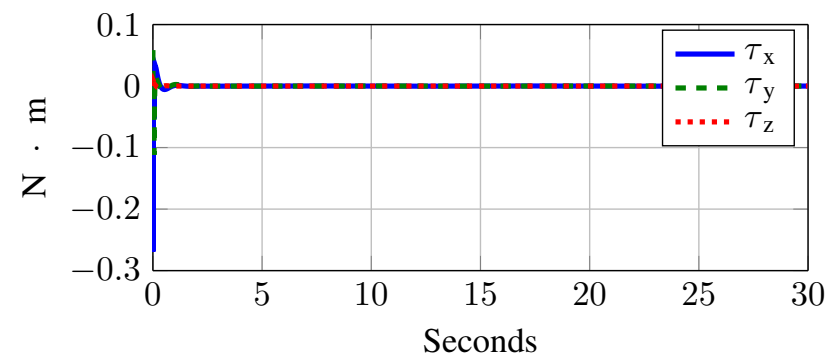

Fig. 11. Torques generated by integral backstepping control face to bigger mass and inertia matrix than modeled

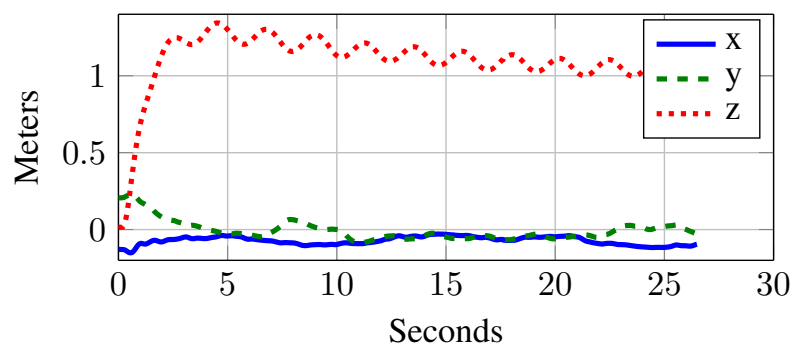

Fig. 12. UAV position under integral backstepping position control

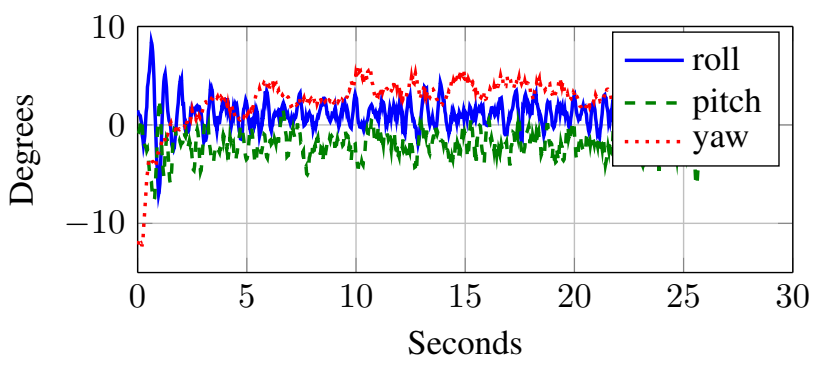

Fig. 13. UAV Euler angles under integral backstepping position control 


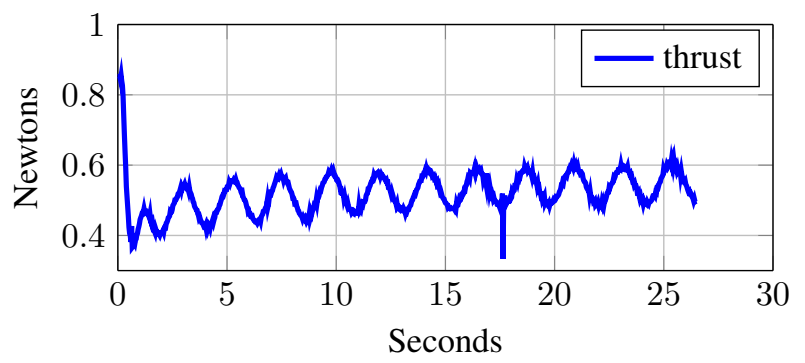

Fig. 14. Thrust generated under integral backstepping position control

\section{Trajectory tracking by the cart}

The parameters used in this simulation were:

$$
\begin{array}{ll}
K_{1 x}=K_{1 y}=K_{1}=4 & K_{2 x}=K_{2 y}=K_{2}=2 \\
M_{1 x}=M_{1 y}=M_{1}=0.25 & M_{2 x}=M_{2 y}=M_{2}=0.25 \\
K_{p x}=K_{p y}=K_{p}=0.3 & K_{q x}=K_{q y}=K_{q}=1 \\
\rho=2 & K_{f}=0.5 \\
K_{f}=0.5 & K_{\text {min }}=0.05
\end{array}
$$

The inertia matrix is supposed to be the same as the UAV drone but here the mass value of the cart $m=0.068$ $\mathrm{kg}$ increased because of wheels mass. The performance on trajectory tracking of the saturation control design in the position model are shown in Figs. 15, 16 and 17. As shown, the cart tracks the circular trajectory over the floor. Then, a good performance in the tracking can be achieved by tunning the parameters of the saturation control.

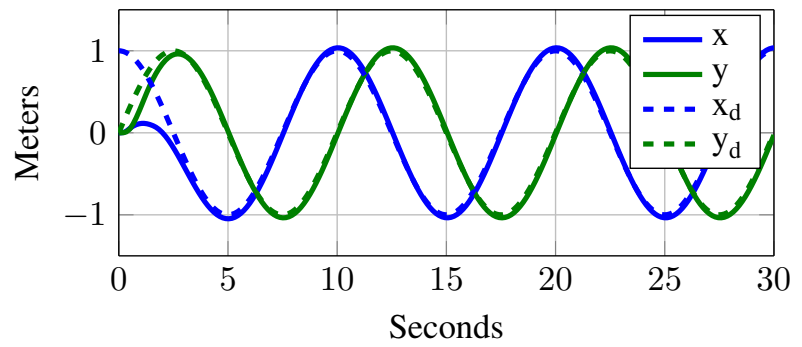

Fig. 15. Tracking with saturation

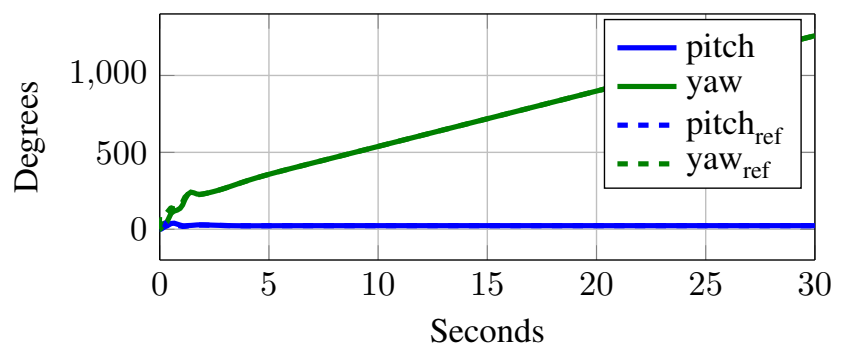

Fig. 16. Euler angles of the cart-drone under saturation control

\section{Experiments results in cart position control}

The testing results of the proposed saturation law are shown in Figs. 18 and 19. As depicted in the figures, the

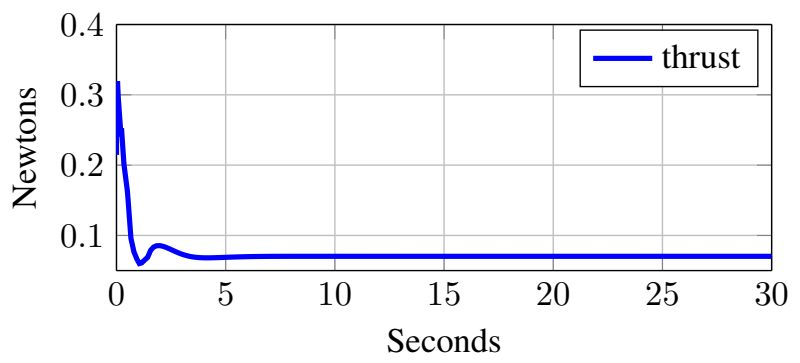

Fig. 17. Thrust generated by the saturation control

reference position $(x, y)=(1,0.5) \mathrm{m}$ can be reached with the proposed law.

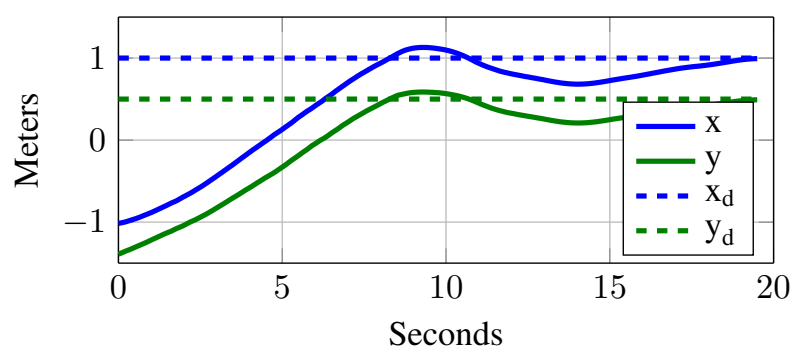

Fig. 18. Cart position generated by applying the saturation control

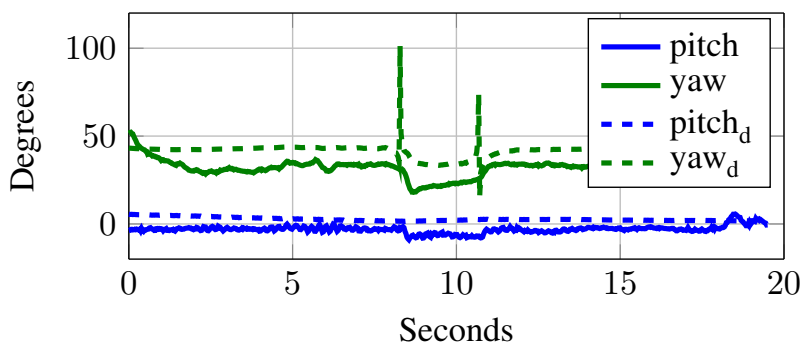

Fig. 19. Pitch and yaw angles generated by applying the saturation control

\section{CONCLUSIONS AND FUTURE WORK}

This paper presented an integral backstepping algorithm applied to an UAV. The vehicle model is reduced and it considers the system as ideal. The integral part of algorithm helped to compensate the uncertainties and dynamics not modeled, showing that, the backstepping algorithm is robust when those uncertainties vary sufficiently slow.

The integral backstepping is helpful when there are uncertainties not modeled, as normally happens. This law has been applied to a mini-drone acting as a UAV and adapted as a cart. The observed results in the experiments were quite satisfactory as a first attempt. In particular, the discharge speed of batteries play an important roll during the control time. The control will force the battery to deliver the same power in order to maintain the required references. 
The next step will be to carry out the trajectory tracking by the vehicle by using the control laws developed in this article. Afterwards, it will be looked a smooth transition between the saturation and backstepping control in order to make a trajectory tracking over floor and in air.

\section{ACKNOWLEDGMENT}

The authors would like to thank B. Boisseau, J. Dumon, J. Minet, G. Hasan and R. Polizzi for fruitful discussions and help in the platform MOCA, the required electronics and the UAV structure designs.

\section{REFERENCES}

[1] C. B. Low, "A Trajectory Tracking Control Design for Fixed-Wing Unmanned Aerial Vehicles," IEEE Conference on Control Applications, pp. 2118-2123, Sep. 2010.

[2] M. B. H. Bouadi and M. Tadjine, "Sliding Mode Control based on Backstepping Approach for an UAV Type-Quadrotor," International Journal of Mechanical, Aerospace, Industrial and Mechatronics Engineering, vol. 1, no. 2, 2007.

[3] M. Tahar, K. Meguenni Zemalache, A. Omari, "Control of an Under-Actuated X4-flyer using Integral Backstepping Controller," PRZEGLAD ELEKTROTECHNICZNY (Electrical Review) ISSN 0033-2097, R. 87 NR 10/2011.

[4] C. Samson, K. Ait-Abderrahim, "Feedback control of a nonholonomic wheeled cart in cartesian space," in Robotics and Automation, 1991. Proceedings., 1991 IEEE International Conference on, no. 2, 1991, pp. 1136-1141.

[5] A. Benalia, M. Djemai, and J.-P. Barbot, "Control of the kinematic car using trajectory generation and the high order sliding mode control," in Systems, Man and Cybernetics, 2003. IEEE International Conference on, vol. 3, Oct 2003, pp. 2455-2460.

[6] C. Wang, Z. Shen, E. Tian, and Q. Zheng, "Multi-smart car control system design and research based on zigbee," in Control and Decision Conference (2014 CCDC), The 26th Chinese, May 2014, pp. 1490-1494.

[7] P. Castillo, R. Lozano and A. E. Dzul, Modelling and Control of Mini-Flying Machines. London: Springer-Verlag, 2005, ch. 2,3.

[8] A. Azzam and X. Wang, "Quad rotor arial robot dynamic modeling and configuration stabilization," in Informatics in Control, Automation and Robotics (CAR), 2010 2nd International Asia Conference on, vol. 1, March 2010, pp. 438-444.

[9] Y. Naidoo, R. Stopforth, and G. Bright, "Development of an uav for search amp; rescue applications," in AFRICON, 2011, Sept 2011, pp. $1-6$.

[10] Zachary Thompson Dydek, "Adaptive control of unmanned aerial systems," Ph.D. dissertation, Massachusetts Institute of Technology, Sept 2010, ch. 3.

[11] A. Brezoescu, R. Lozano, and P. Castillo, "Bank to turn approach for airplane translational motion in unknown wind," in Unmanned Aircraft Systems (ICUAS), 2013 International Conference on, May 2013, pp. 1022-1029.

[12] C. B. Low and Q. S. Ng, "A flexible virtual structure formation keeping control for fixed-wing uavs," in Control and Automation (ICCA), 2011 9th IEEE International Conference on, Dec 2011, pp. 621-626.

[13] Q. Gao, F. Yue, and D. Hu, "Research of stability augmentation hybrid controller for quadrotor uav," in Control and Decision Conference (2014 CCDC), The 26th Chinese, May 2014, pp. 5224-5229.

[14] H. Khebbache, M. Tadjine, "Robust Fuzzy Backstepping Sliding Mode Controller For a Quadrotor Unmanned Aerial Vehicle," CEAI, vol. 15 , no. 2 .

[15] K. Shojaei, A. Shahri, and B. Tabibian, "Adaptive-robust feedback linearizing control of a nonholonomic wheeled mobile robot," in Advanced Intelligent Mechatronics (AIM), 2010 IEEE/ASME International Conference on, July 2010, pp. 497-502.

[16] J. Yang, Z. Cai, Q. Lin, and Y. Wang, "Self-tuning pid control design for quadrotor uav based on adaptive pole placement control," in Chinese Automation Congress (CAC), 2013, Nov 2013, pp. 233-237.
[17] E. Reyes-Valeria, R. Enriquez-Caldera, S. Camacho-Lara, and J. Guichard, "Lqr control for a quadrotor using unit quaternions: Modeling and simulation," in Electronics, Communications and Computing (CONIELECOMP), 2013 International Conference on, March 2013, pp. 172-178.

[18] W. Ren and R. Beard, "Clf-based tracking control for uav kinematic models with saturation constraints," in Decision and Control, 2003. Proceedings. 42nd IEEE Conference on, vol. 4, Dec 2003, pp. 3924-3929 vol.4.

[19] C. Aguilar-Ibanez, J. Martinez-Garcia, and A. Soria-Lopez, "Bounded control based on saturation functions of nonlinear under-actuated mechanical systems: The cart-pendulum system case," in Decision and Control and European Control Conference (CDC-ECC), 2011 50th IEEE Conference on, Dec 2011, pp. 1759-1764.

[20] GIPSA-LAB. MOCA Platform. [Online]. Available: http://www.ctan.org/tex-archive/macros/latex/required/babel/ 